\title{
Catalytic Asymmetric Vinylation and Dienylation of Ketones
}

\author{
Hongmei Li and Patrick J. Walsh* \\ P. Roy and Diane T. Vagelos Laboratories, \\ University of Pennsylvania, Department of Chemistry \\ 231 South 34th Street, Philadelphia, PA 19104-6323.
}

\section{Supporting Information}

Part 1

Table of Contents

Page

$\begin{array}{ll}\text { General Methods } & \text { S2 }\end{array}$

General Procedure for the Preparation of Allylic Alcohols $\quad$ S2

$\begin{array}{ll}\text { Characterization of } \mathbf{S 1}-\mathbf{S 2 3} & \text { S2 }\end{array}$

Conditions for the Determination of Enantiomeric Excess $\quad$ S8

Determination of the Absolute Configuration $\quad$ S9

$\begin{array}{ll}\text { References } & \mathrm{S} 10\end{array}$ 
General Methods. All reactions were carried out under a nitrogen atmosphere with oven-dried glassware. The progress of all reactions was monitored by thin-layer chromatography to ensure the reactions had reached completion. All manipulations involving Schwartz's reagent and dimethylzinc were carried out under an inert atmosphere in a Vacuum Atmospheres drybox with an attached MO-40 Dritrain or by using standard Schlenk or vacuum line techniques. All chemicals were obtained from Aldrich, Acros, or GFS Chemicals unless otherwise specified. All solvents were purchased from Fischer Scientific. Toluene, dichloromethane, diethyl ether and hexanes were dried through activated alumina columns. $\mathrm{Cp}_{2} \mathrm{ZrHCl}$ was prepared according to a modification of literature protocol. ${ }^{1,2}$ Titanium(IV) isopropoxide and all liquid ketone substrates were distilled prior to use. Titanium(IV) isopropoxide (1.4 $\mathrm{M}$ in toluene) and dimethylzinc (1.0 $\mathrm{M}$ in toluene) were prepared and stored in a Vacuum Atmospheres drybox. NMR spectra were obtained on a Brüker $500 \mathrm{MHz}$ Fourier transform spectrometer at the University of Pennsylvania NMR facility. Chemical shifts are reported in units of parts per million downfield from tetramethylsilane. ${ }^{13} \mathrm{C}\left\{{ }^{1} \mathrm{H}\right\}$ NMR spectra were referenced to residual solvent. The infrared spectra were obtained using a Perkin-Elmer 1600 series spectrometer. Thin-layer chromatography was performed on Whatman precoated silica gel 60 F-254 plates and visualized by ultraviolet light or by staining with cerric ammonium molybdate or phosphomolybdic acid solutions. Silica gel (230-400 mesh, Silicycle) was used for air-flashed chromatography.

\section{General Protocol A. Preparation of 2,2-dimethyl-propionic acid 4-hydroxy-4-phenyl-pent-2-enyl ester (S1).}

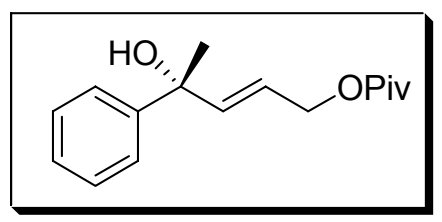

To a suspension of $\mathrm{Cp}_{2} \mathrm{ZrHCl}(155 \mathrm{mg}, 0.60 \mathrm{mmol})$ in $\mathrm{CH}_{2} \mathrm{Cl}_{2}(2.0 \mathrm{~mL})$ under $\mathrm{N}_{2}$ was added 2,2-dimethyl-propionic acid prop-2-ynyl ester (103 mg, $0.60 \mathrm{mmol}$ ) and the reaction mixture was stirred for $10 \mathrm{~min}$ at r.t. after which it was homogeneous. The solvent was removed in vacuo and the residue was dissolved in dry toluene $(2.0 \mathrm{~mL})$, cooled to $-78^{\circ} \mathrm{C}$, treated with $\mathrm{Me}_{2} \mathrm{Zn}(0.30 \mathrm{~mL}, 2.0 \mathrm{M}$ in toluene, $0.60 \mathrm{mmol})$ for $15 \mathrm{~min}$. In another Schlenk flask were mixed ligand $1(13.7 \mathrm{mg}, 0.025 \mathrm{mmol}, 5 \mathrm{~mol} \%)$ in $1.0 \mathrm{~mL}$ toluene and $\mathrm{Ti}\left(\mathrm{O}^{i} \mathrm{Pr}\right)_{4}$ $(0.22 \mathrm{~mL}, 1.4 \mathrm{M}$ toluene solution, $0.30 \mathrm{mmol})$ at r.t. and stirred for $15 \mathrm{~min}$. The resulting solution was added to the Schlenk flask containing the vinylzirconocene and dimethylzinc at $-78^{\circ} \mathrm{C}$. After the addition, the solution was warmed to $0^{\circ} \mathrm{C}$, and acetophenone $(59 \mu \mathrm{L}, 0.50 \mathrm{mmol})$ was added. The reaction mixture was warmed to r.t. and stirred until TLC showed complete consumption of the ketone. The reaction mixture was quenched with saturated $\mathrm{NaHCO}_{3}(5 \mathrm{~mL})$, diluted with EtOAc, filtered through Celite, and the layers were separated. The aqueous layer was extracted with EtOAc $(2 \times 40 \mathrm{~mL})$ and the combined organic layers were washed with brine, dried over $\mathrm{MgSO}_{4}$, and concentrated. The residue was purified by flash chromatography on deactivated silica gel $\left(\mathrm{Et}_{3} \mathrm{~N} / \mathrm{SiO}_{2}=2.5 \% \mathrm{~V} / \mathrm{V}\right.$, hexanes : EtOAc / $90: 10)$ to give the product $\left(110 \mathrm{mg}, 90 \%\right.$ yield, $89 \%$ ee)as an oil: $[\alpha]_{\mathrm{D}}{ }^{20}=+6.7(c$ $\left.0.99, \mathrm{CHCl}_{3}\right) ;{ }^{1} \mathrm{H}$ NMR $\left(\mathrm{C}_{6} \mathrm{D}_{6}, 500 \mathrm{MHz}\right) \delta 1.13(\mathrm{~s}, 9 \mathrm{H}), 1.44(\mathrm{~s}, 3 \mathrm{H}), 4.47(\mathrm{~d}, J=5.8 \mathrm{~Hz}, 2 \mathrm{H}), 5.74-$ $5.78(\mathrm{~m}, 1 \mathrm{H}), 5.90(\mathrm{~d}, J=15.6 \mathrm{~Hz}, 1 \mathrm{H}), 7.06(\mathrm{~m}, 1 \mathrm{H}), 7.15-7.18(\mathrm{~m}, 2 \mathrm{H}), 7.40-7.42(\mathrm{~m}, 2 \mathrm{H}) \mathrm{ppm}$; ${ }^{13} \mathrm{C}\left\{{ }^{1} \mathrm{H}\right\}$ NMR $\left(\mathrm{C}_{6} \mathrm{D}_{6}, 125 \mathrm{MHz}\right) \delta 27.3,29.9,38.8,64.4,73.9,122.5,125.6,127.1,128.4,141.2,147.2$, 177.7 ppm; IR (neat) 3472, 2974, 2875, 1724, 1602, 1482, 1452, $1368 \mathrm{~cm}^{-1}$; HRMS m/z 244.1473 [(M$\left.\mathrm{H}_{2} \mathrm{O}\right)^{+}$; calcd for $\left.\mathrm{C}_{16} \mathrm{H}_{20} \mathrm{O}_{2}: 244.1464\right]$.

\section{Preparation of 5-phenylsulfanyl-2-(3-trifluoromethyl-phenyl)-pent-3-en-2-ol (S2).}

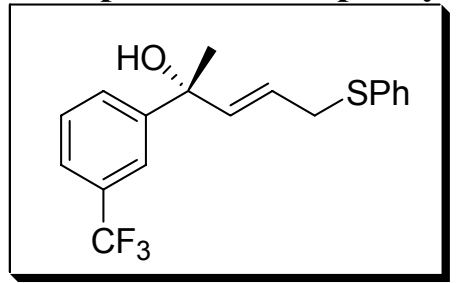

The General Procedure A was applied to acetophenone on a $76 \mu \mathrm{L}(0.5$ mmol) scale. The crude product was purified by column chromatography on deactivated silica gel $\left(\mathrm{Et}_{3} \mathrm{~N} / \mathrm{SiO}_{2}=2.5 \% \mathrm{~V} / \mathrm{V}\right.$, hexanes : EtOAc / 90 : $10)$ to give the product (160 mg, 95\% yield, $88 \%$ ee) as an oil: $[\alpha]_{\mathrm{D}}{ }^{20}=+$ 33.3 (c $\left.0.90, \mathrm{CHCl}_{3}\right) ;{ }^{1} \mathrm{H} \mathrm{NMR}\left(\mathrm{CDCl}_{3}, 500 \mathrm{MHz}\right) \delta 1.55$ (s, 3H), $1.84(\mathrm{br}$, $1 \mathrm{H}), 3.52-3.53(\mathrm{~m}, 2 \mathrm{H}), 5.73-5.74(\mathrm{~m}, 2 \mathrm{H}), 7.18-7.19(\mathrm{~m}, 1 \mathrm{H}), 7.23-7.25$ $(\mathrm{m}, 2 \mathrm{H}), 7.31-7.32(\mathrm{~m}, 2 \mathrm{H}), 7.35-7.41(\mathrm{~m}, 2 \mathrm{H}), 7.48(\mathrm{~d}, J=7.4 \mathrm{~Hz}, 1 \mathrm{H})$, $7.65(\mathrm{~s}, 1 \mathrm{H}) \mathrm{ppm} ;{ }^{13} \mathrm{C}\left\{{ }^{1} \mathrm{H}\right\} \mathrm{NMR}\left(\mathrm{CDCl}_{3}, 125 \mathrm{MHz}\right) \delta 30.2,36.7,74.3,122.3(\mathrm{~m}), 123.5,124.1(\mathrm{~m})$, 125.2, 127.2, 129.0, 129.1, 129.2, 130.8 (q), 131.5, 135.4, 139.8, 147.9 ppm; IR (neat) 3418, 3067, 
2977, 2927, 1584, 1480, $1439 \mathrm{~cm}^{-1}$; HRMS-CI $m / z$ 320.0841[(M-H $\left.\mathrm{H}_{2} \mathrm{O}\right)^{+}$; calcd for $\mathrm{C}_{18} \mathrm{H}_{15} \mathrm{~F}_{3} \mathrm{~S}$ : 320.0846].

\section{Preparation of 3-methyl-1-phenyl-non-4-en-1-yn-3-ol (S3).}

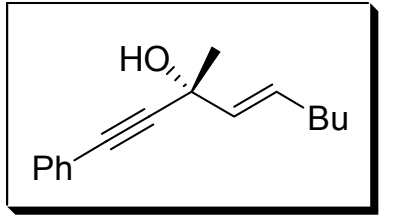

The General Procedure A was applied to 4-phenyl-but-3-yn-2-one on a 73 $\mu \mathrm{L}(0.5 \mathrm{mmol}) \mathrm{scale}$. The crude product was purified by column chromatography on deactivated silica gel $\left(\mathrm{Et}_{3} \mathrm{~N} / \mathrm{SiO}_{2}=2.5 \% \mathrm{~V} / \mathrm{V}\right.$, hexanes : EtOAc / $95: 5)$ to give the product ( $85 \mathrm{mg}, 81 \%$ yield, $79 \%$ ee) as an oil: $[\alpha]_{\mathrm{D}}{ }^{20}=-8.9\left(c 0.83, \mathrm{CHCl}_{3}\right) ;{ }^{1} \mathrm{H}$ NMR $\left(\mathrm{C}_{6} \mathrm{D}_{6}, 500 \mathrm{MHz}\right) \delta 0.83(\mathrm{t}, J=7.1 \mathrm{~Hz}$, $3 \mathrm{H}), 1.22-1.27(\mathrm{~m}, 4 \mathrm{H}), 1.95(\mathrm{~s}, 3 \mathrm{H}), 1.94-1.96(\mathrm{~m}, 2 \mathrm{H}), 5.71(\mathrm{~d}, J=15.3 \mathrm{~Hz}, 1 \mathrm{H}), 6.10(\mathrm{dt}, J=15.3$, $6.8 \mathrm{~Hz}, 1 \mathrm{H}), 6.96-7.00(\mathrm{~m}, 3 \mathrm{H}), 7.41-7.44(\mathrm{~m}, 2 \mathrm{H}) \mathrm{ppm} ;{ }^{13} \mathrm{C}\left\{{ }^{1} \mathrm{H}\right\} \mathrm{NMR}\left(\mathrm{C}_{6} \mathrm{D}_{6}, 125 \mathrm{MHz}\right) \delta 14.1,22.6$, 31.0, 31.6, 31.9, 68.3, 84.6, 93.2, 123.6, 128.4, 128.6, 130.0, 132.0, 135.2 ppm; IR (neat) 3353, 2957, $2928,2861,1668,1598,1490,1446 \mathrm{~cm}^{-1}$; HRMS $m / z 210.1416\left[\left(\mathrm{M}-\mathrm{H}_{2} \mathrm{O}\right)^{+}\right.$; calcd for $\mathrm{C}_{16} \mathrm{H}_{18}$ : 210.1408].

\section{Preparation of 2-bromo-1-hex-1-enyl-cyclohex-2-enol (S4).}

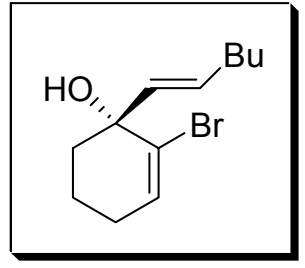

The General Procedure A was applied to 2-bromo-cyclohex-2-enone on a $88 \mathrm{mg}$ $(0.5 \mathrm{mmol})$ scale. The crude product was purified by column chromatography on deactivated silica gel $\left(\mathrm{Et}_{3} \mathrm{~N} / \mathrm{SiO}_{2}=2.5 \% \mathrm{~V} / \mathrm{V}\right.$, hexanes : EtOAc / $\left.97: 3\right)$ to give the product (50 mg, $42 \%$ yield, $92 \%$ ee) as an oil: $[\alpha]_{\mathrm{D}}{ }^{20}=-0.1\left(c 0.67, \mathrm{CHCl}_{3}\right) ;{ }^{\mathrm{l}} \mathrm{H}$ $\operatorname{NMR}\left(\mathrm{CDCl}_{3}, 500 \mathrm{MHz}\right) \delta 0.89(\mathrm{t}, J=7.2 \mathrm{~Hz}, 3 \mathrm{H}), 1.31-1.38(\mathrm{~m}, 4 \mathrm{H}), 1.61-1.64$ $(\mathrm{m}, 1 \mathrm{H}), 1.65-1.66(\mathrm{~m}, 1 \mathrm{H}), 1.90-1.94(\mathrm{~m}, 2 \mathrm{H}), 2.04-2.12(\mathrm{~m}, 4 \mathrm{H}), 2.28(\mathrm{~s}, 1 \mathrm{H}$ from $\mathrm{OH}), 5.44(\mathrm{~d}, J=15.5 \mathrm{~Hz}, 1 \mathrm{H}), 5.70(\mathrm{dt}, J=15.5,6.9 \mathrm{~Hz}, 1 \mathrm{H}), 6.20(\mathrm{t}, J=4.1 \mathrm{~Hz}, 1 \mathrm{H}) \mathrm{ppm} ;{ }^{13} \mathrm{C}\left\{{ }^{1} \mathrm{H}\right\}$ $\mathrm{NMR}\left(\mathrm{CDCl}_{3}, 125 \mathrm{MHz}\right) \delta 14.3,19.4,22.5,28.3,31.7,32.2,37.9,74.4,129.9,131.9,132.7,134.2$

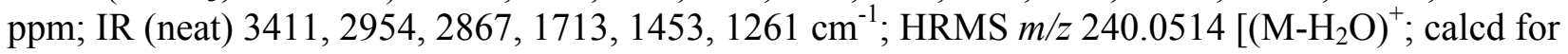
$\left.\mathrm{C}_{12} \mathrm{H}_{17} \mathrm{Br}: 240.0513\right]$.

\section{Preparation of 4-cyclohex-1-enyl-2-phenyl-but-3-en-2-ol (S5).}

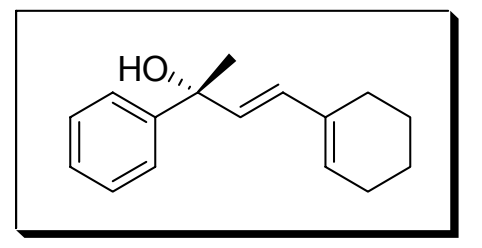

The General Procedure A was applied to acetophenone on a $59 \mu \mathrm{L}(0.5$ $\mathrm{mmol})$ scale. The crude product was purified by flash chromatography on deactivated silica gel $\left(\mathrm{Et}_{3} \mathrm{~N} / \mathrm{SiO}_{2}=2.5 \% \mathrm{~V} / \mathrm{V}\right.$, hexanes : EtOAc / $\left.96: 4\right)$ to give the product (101 mg, $89 \%$ yield, $89 \%$ ee) as an oil: $[\alpha]_{\mathrm{D}}{ }^{20}=-1.8(\mathrm{c}$ $\left.1.37, \mathrm{CHCl}_{3}\right) ;{ }^{1} \mathrm{H}$ NMR $\left(\mathrm{C}_{6} \mathrm{D}_{6}, 500 \mathrm{MHz}\right) \delta 1.41-1.52(\mathrm{~m}, 4 \mathrm{H}), 1.54(\mathrm{~s}$, $3 \mathrm{H}), 1.95-1.97(\mathrm{~m}, 4 \mathrm{H}), 5.64(\mathrm{~m}, 1 \mathrm{H}), 5.82(\mathrm{~d}, J=15.9 \mathrm{~Hz}, 1 \mathrm{H}), 6.32(\mathrm{~d}, J$ $=15.9 \mathrm{~Hz}, 1 \mathrm{H}), 7.07-7.10(\mathrm{~m}, 1 \mathrm{H}), 7.18-7.21(\mathrm{~m}, 2 \mathrm{H}), 7.50-7.52(\mathrm{~m}, 2 \mathrm{H}) \mathrm{ppm} ;{ }^{13} \mathrm{C}\left\{{ }^{1} \mathrm{H}\right\} \mathrm{NMR}\left(\mathrm{C}_{6} \mathrm{D}_{6}\right.$, $125 \mathrm{MHz}) \delta 22.9,23.0,25.0,26.2,30.3,74.6,125.8,126.9,128.4,129.7,131.5,133.3,135.5,148.2$ ppm; IR (neat) 3424, 3026, 2929, 2860, 1714, 1669, 1492, $1445 \mathrm{~cm}^{-1}$; HRMS m/z 228.1509 [M+calcd for $\left.\mathrm{C}_{16} \mathrm{H}_{20} \mathrm{O}: 228.1514\right]$.

\section{Preparation of 4-cyclohex-1-enyl-2-m-toly-but-3-en-2-ol (S6).}

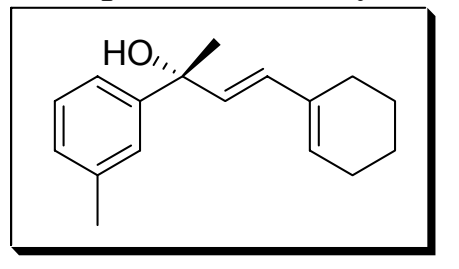

The General Procedure A was applied to 3'-methyl-acetophenone on a 55 $\mu \mathrm{L}(0.4 \mathrm{mmol})$ scale. The crude product was purified by column chromatography on deactivated silica gel $\left(\mathrm{Et}_{3} \mathrm{~N} / \mathrm{SiO}_{2}=2.5 \% \mathrm{~V} / \mathrm{V}\right.$, hexanes : EtOAc / 96 : 4) to give the product ( $89 \mathrm{mg}, 92 \%$ yield, $92 \%$ ee) as an oil: $[\alpha]_{\mathrm{D}}{ }^{20}=+4.2\left(c 1.10, \mathrm{CHCl}_{3}\right) ;{ }^{1} \mathrm{H}$ NMR $\left(\mathrm{C}_{6} \mathrm{D}_{6}, 500 \mathrm{MHz}\right) \delta 1.38-1.50(\mathrm{~m}$, $4 \mathrm{H}), 1.59(\mathrm{~s}, 3 \mathrm{H}), 1.95-1.98(\mathrm{~m}, 4 \mathrm{H}), 2.17(\mathrm{~s}, 3 \mathrm{H}), 5.66(\mathrm{~m}, 1 \mathrm{H}), 5.86(\mathrm{~d}, J$ $=15.9 \mathrm{~Hz}, 1 \mathrm{H}), 6.37(\mathrm{~d}, J=15.9 \mathrm{~Hz}, 1 \mathrm{H}), 6.94(\mathrm{~d}, J=7.4 \mathrm{~Hz}, 1 \mathrm{H}), 7.16(\mathrm{dd}, J=7.8,7.4 \mathrm{~Hz}, 1 \mathrm{H}), 7.36$ $(\mathrm{d}, J=7.8 \mathrm{~Hz}, 1 \mathrm{H}), 7.43(\mathrm{~s}, 1 \mathrm{H}) \mathrm{ppm} ;{ }^{13} \mathrm{C}\left\{{ }^{1} \mathrm{H}\right\} \mathrm{NMR}\left(\mathrm{C}_{6} \mathrm{D}_{6}, 125 \mathrm{MHz}\right) \delta 21.7,22.9,23.0,25.0,26.2$, 30.4, 74.5, 123.0, 126.5, 127.7, 128.4, 129.7, 131.4, 133.5, 135.5, 137.8, 148.3 ppm; IR (neat) 3410 , 2932, 2859, 1676, 1606, 1485, 1451, $1365 \mathrm{~cm}^{-1}$; HRMS m/z 224.1573 [(M-H $\left.\mathrm{H}_{2} \mathrm{O}\right)^{+}$; calcd for $\mathrm{C}_{17} \mathrm{H}_{20}$ : 224.1566]. 


\section{Preparation of 4-cyclohex-1-enyl-2-(3-trifluoromethyl-phenyl)-but-3-en-2-ol (S7).}

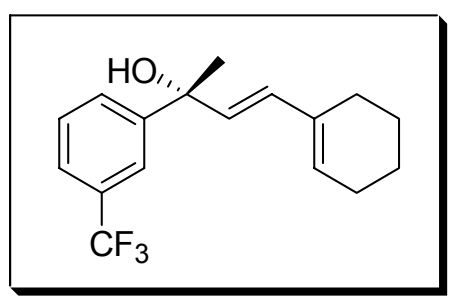

The General Procedure A was applied to 3'-

(trifluoromethyl)acetophenone on a $76 \mu \mathrm{L}(0.5 \mathrm{mmol})$ scale. The crude product was purified by column chromatography on deactivated silica gel $\left(\mathrm{Et}_{3} \mathrm{~N} / \mathrm{SiO}_{2}=2.5 \% \mathrm{~V} / \mathrm{V}\right.$, hexanes : EtOAc / $\left.96: 4\right)$ to give the product $(138 \mathrm{mg}, 93 \%$ yield, $87 \%$ ee $)$ as an oil: $[\alpha]_{\mathrm{D}}{ }^{20}=+3.5\left(c 0.99, \mathrm{CHCl}_{3}\right) ;{ }^{1} \mathrm{H}$ NMR $\left(\mathrm{C}_{6} \mathrm{D}_{6}, 500 \mathrm{MHz}\right) \delta 1.42(\mathrm{~s}, 3 \mathrm{H}), 1.43-1.50(\mathrm{~m}, 4 \mathrm{H}), 1.91-1.95(\mathrm{~m}$, $4 \mathrm{H}), 5.62(\mathrm{~m}, 1 \mathrm{H}), 5.67(\mathrm{~d}, J=15.9 \mathrm{~Hz}, 1 \mathrm{H}), 6.22(\mathrm{~d}, J=15.9 \mathrm{~Hz}, 1 \mathrm{H})$, $7.00(\mathrm{dd}, J=7.8,7.7 \mathrm{~Hz}, 1 \mathrm{H}), 7.30(\mathrm{~d}, J=7.7 \mathrm{~Hz}, 1 \mathrm{H}), 7.44(\mathrm{~d}, J=7.8 \mathrm{~Hz}, 1 \mathrm{H}), 7.96(\mathrm{~s}, 1 \mathrm{H}) \mathrm{ppm}$; ${ }^{13} \mathrm{C}\left\{{ }^{1} \mathrm{H}\right\} \operatorname{NMR}\left(\mathrm{C}_{6} \mathrm{D}_{6}, 125 \mathrm{MHz}\right) \delta 22.7,22.8,24.8,26.2,30.3,74.2,122.3(\mathrm{~m}), 123.7(\mathrm{~m}), 126.3,128.9$, 129.4, 130.6, 130.7 (q), 132.1, 132.3, 135.2, 149.4 ppm; IR (neat) 3425, 2934, 2864, 1685, 1666, 1617, 1440, $1329 \mathrm{~cm}^{-1}$; HRMS-CI $\mathrm{m} / z 278.1285$ [(M-H $\left.\mathrm{H}_{2} \mathrm{O}\right)^{+}$; calcd for $\left.\mathrm{C}_{17} \mathrm{H}_{17} \mathrm{~F}_{3}: 278.1282\right]$.

\section{Preparation of 6-methyl-2-m-tolyl-dodeca-3, 5-dien-2-ol (S8).}

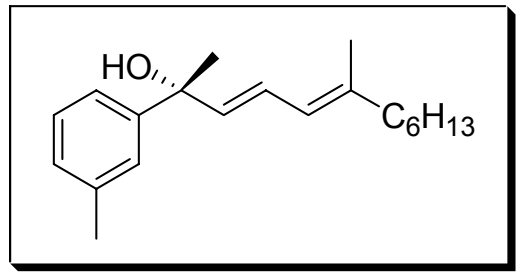

The General Procedure A was applied to 3'-methyl-acetophenone on a $55 \mu \mathrm{L}(0.4 \mathrm{mmol})$ scale. The crude product was purified by column chromatography on deactivated silica gel $\left(\mathrm{Et}_{3} \mathrm{~N} / \mathrm{SiO}_{2}=2.5 \% \mathrm{~V} / \mathrm{V}\right.$, hexanes : EtOAc / $96: 4$ ) to give the product ( $78 \mathrm{mg}, 68 \%$ yield, $89 \%$ ee) as an oil: $[\alpha]_{\mathrm{D}}{ }^{20}=-8.9\left(c 0.91, \mathrm{CHCl}_{3}\right) ;{ }^{1} \mathrm{H}$ NMR $\left(\mathrm{C}_{6} \mathrm{D}_{6}, 500 \mathrm{MHz}\right)$ $\delta 0.88(\mathrm{t}, J=6.8 \mathrm{~Hz}, 3 \mathrm{H}), 1.23-1.28(\mathrm{~m}, 6 \mathrm{H}), 1.34-1.39(\mathrm{~m}, 2 \mathrm{H}), 1.57$

$(\mathrm{s}, 3 \mathrm{H}), 1.65(\mathrm{~s}, 3 \mathrm{H}), 1.99(\mathrm{t}, J=7.4 \mathrm{~Hz}, 2 \mathrm{H}), 2.16(\mathrm{~s}, 3 \mathrm{H}), 5.89(\mathrm{~d}, J=15.2 \mathrm{~Hz}, 1 \mathrm{H}), 5.94(\mathrm{~d}, J=10.9$ $\mathrm{Hz}, 1 \mathrm{H}), 6.66$ (dd, $J=15.2,10.9 \mathrm{~Hz}, 1 \mathrm{H}), 6.93(\mathrm{~d}, J=7.5 \mathrm{~Hz}, 1 \mathrm{H}), 7.14(\mathrm{~m}, 1 \mathrm{H}), 7.32$ (d, $J=7.8 \mathrm{~Hz}$, $1 \mathrm{H}), 7.39(\mathrm{~s}, 1 \mathrm{H}) \mathrm{ppm} ;{ }^{13} \mathrm{C}\left\{{ }^{1} \mathrm{H}\right\}$ NMR $\left(\mathrm{C}_{6} \mathrm{D}_{6}, 125 \mathrm{MHz}\right) \delta 14.3,16.7,21.6,23.0,28.3,29.4,30.4,32.2$, $40.3,74.5,122.9,124.3,125.0,126.4,127.7,128.4,137.7,138.9,139.1,148.1$ ppm; IR (neat) 3406 , $2925,2857,1686,1605,1455,1373 \mathrm{~cm}^{-1}$; HRMS $m / z 268.2182\left[\left(\mathrm{M}-\mathrm{H}_{2} \mathrm{O}\right)^{+}\right.$; calcd for $\mathrm{C}_{20} \mathrm{H}_{28}$ : 268.2191].

\section{Preparation of 6-methyl-2-(3-trifluoromethyl-phenyl)-dodeca-3, 5-dien-2-ol (S9).}

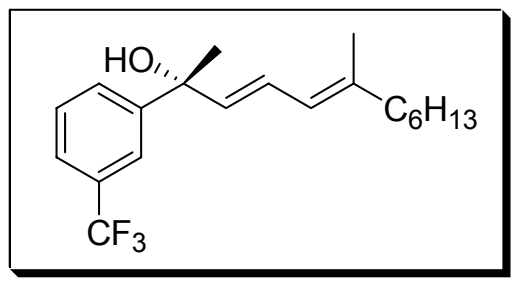

The General Procedure A was applied to 3'-

(trifluoromethyl)acetophenone on a $61 \mu \mathrm{L}(0.5 \mathrm{mmol})$ scale. The crude product was purified by column chromatography on deactivated silica gel $\left(\mathrm{Et}_{3} \mathrm{~N} / \mathrm{SiO}_{2}=2.5 \% \mathrm{~V} / \mathrm{V}\right.$, hexanes : EtOAc $\left./ 96: 4\right)$ to give the product ( $150 \mathrm{mg}, 88 \%$ yield, $94 \%$ ee) as an oil: $[\alpha]_{\mathrm{D}}{ }^{20}=+3.0(c 0.99$, $\left.\mathrm{CHCl}_{3}\right) ;{ }^{1} \mathrm{H}$ NMR $\left(\mathrm{C}_{6} \mathrm{D}_{6}, 500 \mathrm{MHz}\right) \delta 0.88(\mathrm{t}, J=6.8 \mathrm{~Hz}, 3 \mathrm{H}), 1.23-$ $1.28(\mathrm{~m}, 6 \mathrm{H}), 1.35-1.39(\mathrm{~m}, 5 \mathrm{H}), 1.62(\mathrm{~s}, 3 \mathrm{H}), 1.97(\mathrm{t}, J=7.4 \mathrm{~Hz}, 2 \mathrm{H})$, $5.66(\mathrm{~d}, J=15.2 \mathrm{~Hz}, 1 \mathrm{H}), 5.86(\mathrm{~d}, J=10.8 \mathrm{~Hz}, 1 \mathrm{H}), 6.54(\mathrm{dd}, J=15.2,10.8 \mathrm{~Hz}, 1 \mathrm{H}), 6.95(\mathrm{dd}, J=7.8$, $7.7 \mathrm{~Hz}, 1 \mathrm{H}), 7.82(\mathrm{~d}, J=7.7 \mathrm{~Hz}, 1 \mathrm{H}), 7.40(\mathrm{~d}, J=7.8 \mathrm{~Hz}, 1 \mathrm{H}), 7.93(\mathrm{~s}, 1 \mathrm{H}) \mathrm{ppm} ;{ }^{13} \mathrm{C}\left\{{ }^{1} \mathrm{H}\right\} \mathrm{NMR}\left(\mathrm{C}_{6} \mathrm{D}_{6}\right.$, $125 \mathrm{MHz}) \delta 14.3,16.6,23.0,28.1,29.4,30.1,32.1,40.2,74.1,122.3(\mathrm{~m}), 123.7(\mathrm{~m}), 124.5,125.1$, 126.2, 128.8, 129.2, 130.7 (q), 137.4, 140.1, 149.2 ppm; IR (neat) 3378, 2928, 2858, 1653, 1444, 1328 $\mathrm{cm}^{-1}$; HRMS $m / z$ 322.1905 [(M-H $\left.\mathrm{H}_{2} \mathrm{O}\right)^{+}$; calcd for $\left.\mathrm{C}_{20} \mathrm{H}_{25} \mathrm{~F}_{3}: 322.1908\right]$.

\section{Preparation of 5-methyl-2-phenyl-hexa-3, 5-dien-2-ol (S10).}

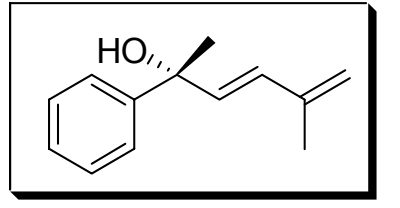

The General Procedure A was applied to acetophenone on a $59 \mu \mathrm{L}(0.5$ mmol) scale. The crude product was purified by column chromatography on deactivated silica gel $\left(\mathrm{Et}_{3} \mathrm{~N} / \mathrm{SiO}_{2}=2.5 \% \mathrm{~V} / \mathrm{V}\right.$, hexanes : EtOAc $\left./ 95: 5\right)$ to give the product $\left(93 \mathrm{mg}, 99 \%\right.$ yield, $93 \%$ ee) as an oil: $[\alpha]_{\mathrm{D}}{ }^{20}=-12.2(c 0.72$, $\left.\mathrm{CHCl}_{3}\right) ;{ }^{1} \mathrm{H}$ NMR $\left(\mathrm{C}_{6} \mathrm{D}_{6}, 500 \mathrm{MHz}\right) \delta 1.50(\mathrm{~s}, 3 \mathrm{H}), 1.67(\mathrm{~s}, 3 \mathrm{H}), 1.97(\mathrm{br}, 1 \mathrm{H})$, $4.90(\mathrm{~s}, 1 \mathrm{H}), 4.94(\mathrm{~s}, 1 \mathrm{H}), 5.88(\mathrm{~d}, J=15.9 \mathrm{~Hz}, 1 \mathrm{H}), 6.41(\mathrm{~d}, J=15.9 \mathrm{~Hz}, 1 \mathrm{H}), 7.06(\mathrm{t}, J=7.2 \mathrm{~Hz}, 1 \mathrm{H})$, $7.17(\mathrm{dd}, J=7.8,7.5 \mathrm{~Hz}, 2 \mathrm{H}), 7.44(\mathrm{~d}, J=7.7 \mathrm{~Hz}, 2 \mathrm{H}) \mathrm{ppm} ;{ }^{13} \mathrm{C}\left\{{ }^{1} \mathrm{H}\right\} \mathrm{NMR}\left(\mathrm{C}_{6} \mathrm{D}_{6}, 125 \mathrm{MHz}\right) \delta 18.7$, 30.0, 74.4, 116.9, 125.6, 127.0, 128.4, 130.5, 137.2, 141.7, 147.6 ppm; IR (neat) 3409, 3028, 2976, $2930,1886,1676,1607,1446,1367 \mathrm{~cm}^{-1}$; HRMS $m / z 170.1090\left[\left(\mathrm{M}-\mathrm{H}_{2} \mathrm{O}\right)^{+}\right.$; calcd for $\mathrm{C}_{13} \mathrm{H}_{14}$ : 170.1095]. 


\section{Preparation of 5-methyl-2-(3-trifluoromethyl-phenyl)-hexa-3, 5-dien-2-ol (S11).}

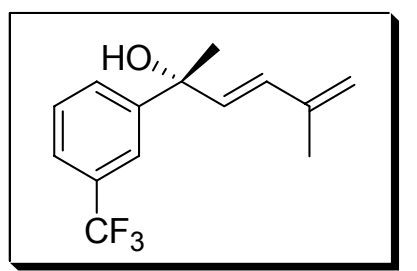

The General Procedure A was applied to 3'-(trifluoromethyl)acetophenone on a $76 \mu \mathrm{L}(0.5 \mathrm{mmol})$ scale. The crude product was purified by column chromatography on deactivated silica gel $\left(\mathrm{Et}_{3} \mathrm{~N} / \mathrm{SiO}_{2}=2.5 \% \mathrm{~V} / \mathrm{V}\right.$, hexanes : EtOAc / $95: 5)$ to give the product (107 $\mathrm{mg}, 84 \%$ yield, $90 \%$ ee) as an oil: $[\alpha]_{\mathrm{D}}^{20}=+5.6\left(c 1.00, \mathrm{CHCl}_{3}\right) ;{ }^{1} \mathrm{H}$ NMR $\left(\mathrm{C}_{6} \mathrm{D}_{6}, 500 \mathrm{MHz}\right) \delta 1.35(\mathrm{~s}, 3 \mathrm{H}), 1.64$ $(\mathrm{s}, 3 \mathrm{H}), 4.91(\mathrm{~s}, 1 \mathrm{H}), 4.93(\mathrm{~s}, 1 \mathrm{H}), 5.72(\mathrm{~d}, J=15.9 \mathrm{~Hz}, 1 \mathrm{H}), 6.31(\mathrm{~d}, J=15.9$ $\mathrm{Hz}, 1 \mathrm{H}), 6.98(\mathrm{t}, J=7.8 \mathrm{~Hz}, 1 \mathrm{H}), 7.29(\mathrm{~d}, J=7.6 \mathrm{~Hz}, 1 \mathrm{H}), 7.37(\mathrm{~d}, J=7.8 \mathrm{~Hz}$, $1 \mathrm{H}), 7.91(\mathrm{~s}, 1 \mathrm{H}) \mathrm{ppm} ;{ }^{13} \mathrm{C}\left\{{ }^{1} \mathrm{H}\right\}$ NMR $\left(\mathrm{C}_{6} \mathrm{D}_{6}, 125 \mathrm{MHz}\right) \delta 18.9,29.8,74.0,117.6,122.3(\mathrm{~m}), 123.9(\mathrm{~m})$, 126.2, 128.9, 129.3, 130.8 (q), 131.3, 136.0, 141.5, 148.9 ppm; IR (neat) 3374, 2979, 2934, 1797, 1609, $1440,1328 \mathrm{~cm}^{-1}$; HRMS m/z 256.1062 [M $\mathrm{M}^{+}$; calcd for $\left.\mathrm{C}_{14} \mathrm{H}_{15} \mathrm{~F}_{3} \mathrm{O}: 256.1075\right]$.

\section{Preparation of 2-(2-bromo-phenyl)-4-cyclohex-1-enyl-but-3-en-2-ol (S12).}

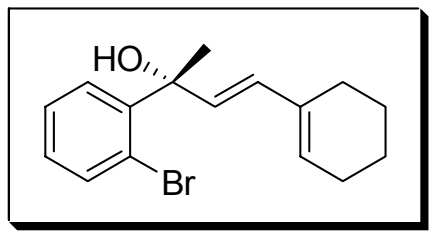

The General Procedure A was applied to 2'-bromo-acetophenoneon a 67 $\mu \mathrm{L}(0.5 \mathrm{mmol})$ scale. The crude product was purified by column chromatography on deactivated silica gel $\left(\mathrm{Et}_{3} \mathrm{~N} / \mathrm{SiO}_{2}=2.5 \% \mathrm{~V} / \mathrm{V}\right.$, hexanes : EtOAc / $96: 4)$ to give the product ( $65 \mathrm{mg}, 42 \%$ yield, $87 \%$ ee) as an oil: $[\alpha]_{\mathrm{D}}{ }^{20}=-20.8\left(c 1.55, \mathrm{CHCl}_{3}\right) ;{ }^{1} \mathrm{H}$ NMR $\left(\mathrm{C}_{6} \mathrm{D}_{6}, 500 \mathrm{MHz}\right) \delta 1.39-1.44(\mathrm{~m}$, $2 \mathrm{H}), 1.47-1.52(\mathrm{~m}, 2 \mathrm{H}), 1.79(\mathrm{~s}, 3 \mathrm{H}), 1.92-1.93(\mathrm{~m}, 2 \mathrm{H}), 2.02-2.05(\mathrm{~m}, 2 \mathrm{H})$, $5.57(\mathrm{~m}, 1 \mathrm{H}), 6.09(\mathrm{~d}, J=16.0 \mathrm{~Hz}, 1 \mathrm{H}), 6.27(\mathrm{~d}, J=16.0 \mathrm{~Hz}, 1 \mathrm{H}), 6.70(\mathrm{~m}, 1 \mathrm{H}), 7.01(\mathrm{~m}, 1 \mathrm{H}), 7.40(\mathrm{~m}$, $1 \mathrm{H}), 7.83(\mathrm{~m}, 1 \mathrm{H}) \mathrm{ppm} ;{ }^{13} \mathrm{C}\left\{{ }^{1} \mathrm{H}\right\}$ NMR $\left(\mathrm{C}_{6} \mathrm{D}_{6}, 125 \mathrm{MHz}\right) \delta 22.8,22.9,24.9,26.2,28.6,75.2,121.7$, 127.5, 128.4, 128.7, 130.0, 132.0, 133.6, 135.1, 135.6, 146.4 ppm; IR (neat) 3426, 2928, 2859, 1700, 1664, 1459, $1429 \mathrm{~cm}^{-1}$; HRMS $m / z 288.0503$ [(M-H $\left.\mathrm{H}_{2} \mathrm{O}\right)^{+}$; calcd for $\left.\mathrm{C}_{16} \mathrm{H}_{17} \mathrm{Br}: 288.0513\right]$.

\section{Preparation of 3-(3-chloro-phenyl)-1-cyclohex-1-enyl-pent-1-en-3-ol (S13).}

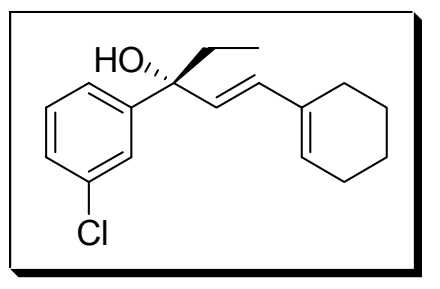

The General Procedure A was applied to 3'-chloropropiophenone on a 84 $\mathrm{mg}(0.5 \mathrm{mmol}) \mathrm{scale}$. The crude product was purified by column chromatography on deactivated silica gel $\left(\mathrm{Et}_{3} \mathrm{~N} / \mathrm{SiO}_{2}=2.5 \% \mathrm{~V} / \mathrm{V}\right.$, hexanes : EtOAc / $97: 3$ ) to give the product ( $96 \mathrm{mg}, 85 \%$ yield, $89 \%$ ee) as an oil: $[\alpha]_{\mathrm{D}}{ }^{20}=-1.9\left(c 0.82, \mathrm{CHCl}_{3}\right) ;{ }^{1} \mathrm{H}$ NMR $\left(\mathrm{C}_{6} \mathrm{D}_{6}, 500 \mathrm{MHz}\right) \delta 0.75(\mathrm{t}, J=7.3$ $\mathrm{Hz}, 3 \mathrm{H}), 1.41-1.49(\mathrm{~m}, 4 \mathrm{H}), 1.66-1.72(\mathrm{~m}, 2 \mathrm{H}), 1.93-1.95(\mathrm{~m}, 4 \mathrm{H}), 5.63(\mathrm{~m}$, $1 \mathrm{H}), 5.69(\mathrm{~d}, J=15.9 \mathrm{~Hz}, 1 \mathrm{H}), 6.26(\mathrm{~d}, J=15.9 \mathrm{~Hz}, 1 \mathrm{H}), 6.90(\mathrm{dd}, J=7.9$, $7.8 \mathrm{~Hz}, 1 \mathrm{H}), 7.06(\mathrm{~d}, J=7.8 \mathrm{~Hz}, 1 \mathrm{H}), 7.18(\mathrm{~d}, J=7.8 \mathrm{~Hz}, 1 \mathrm{H}), 7.63(\mathrm{~s}, 1 \mathrm{H}) \mathrm{ppm} ;{ }^{13} \mathrm{C}\left\{{ }^{1} \mathrm{H}\right\} \mathrm{NMR}\left(\mathrm{C}_{6} \mathrm{D}_{6}\right.$, $125 \mathrm{MHz}) \delta 8.1,22.8,22.9,24.9,26.2,35.5,76.7,124.3,126.4,126.9,129.6,130.2,131.6,132.5$, 134.6, 135.3, 149.4 ppm; IR (neat) 3438, 3026, 2933, 2878, 1647, 1593, 1571, $1467 \mathrm{~cm}^{-1}$; HRMS-CI $m / z 258.1167\left[\left(\mathrm{M}-\mathrm{H}_{2} \mathrm{O}\right)^{+}\right.$; calcd for $\left.\mathrm{C}_{17} \mathrm{H}_{19} \mathrm{Cl}: 258.1175\right]$.

\section{Preparation of 3-(4-chloro-phenyl)-1-cyclohex-1-enyl-pent-1-en-3-ol (S14).}

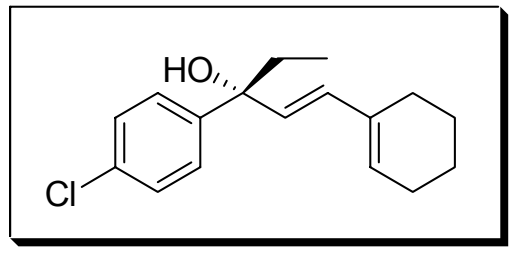

The General Procedure A was applied to 4'-chloropropiophenone on a $84 \mathrm{mg}(0.5 \mathrm{mmol})$ scale. The crude product was purified by column chromatography on deactivated silica gel $\left(\mathrm{Et}_{3} \mathrm{~N} / \mathrm{SiO}_{2}=2.5 \% \mathrm{~V} / \mathrm{V}\right.$, hexanes : EtOAc / $97: 3$ ) to give the product ( $91 \mathrm{mg}, 80 \%$ yield, $79 \%$ ee) an oil: $[\alpha]_{\mathrm{D}}{ }^{20}=-12.6\left(c 0.98, \mathrm{CHCl}_{3}\right) ;{ }^{1} \mathrm{H} \operatorname{NMR}\left(\mathrm{C}_{6} \mathrm{D}_{6}, 500 \mathrm{MHz}\right) \delta$ $0.77(\mathrm{t}, J=7.3 \mathrm{~Hz}, 3 \mathrm{H}), 1.43-1.46(\mathrm{~m}, 2 \mathrm{H}), 1.48-1.51(\mathrm{~m}, 2 \mathrm{H}), 1.67-$ $1.76(\mathrm{~m}, 2 \mathrm{H}), 1.95-1.97(\mathrm{~m}, 4 \mathrm{H}), 5.65(\mathrm{~m}, 1 \mathrm{H}), 5.70(\mathrm{~d}, J=15.9 \mathrm{~Hz}, 1 \mathrm{H}), 6.25(\mathrm{~d}, J=15.9 \mathrm{~Hz}, 1 \mathrm{H})$, $7.16(\mathrm{~d}, J=8.6 \mathrm{~Hz}, 2 \mathrm{H}), 7.20(\mathrm{~d}, J=8.6 \mathrm{~Hz}, 2 \mathrm{H}) \mathrm{ppm} ;{ }^{13} \mathrm{C}\left\{{ }^{1} \mathrm{H}\right\} \mathrm{NMR}\left(\mathrm{C}_{6} \mathrm{D}_{6}, 125 \mathrm{MHz}\right) \delta 8.1,22.8$, 22.9, 25.0, 26.2, 35.5, 76.6, 127.7, 128.4, 130.1, 132.3, 132.7, 134.6, 135.3, 145.4 ppm; IR (neat) 3431, 2934, 2862, 1681, 1591, 1489, $1455 \mathrm{~cm}^{-1}$; HRMS $m / z 258.1180\left[\left(\mathrm{M}-\mathrm{H}_{2} \mathrm{O}\right)^{+}\right.$; calcd for $\mathrm{C}_{17} \mathrm{H}_{19} \mathrm{Cl}$ : 258.1175].

Preparation of 4-cyclohex-1-enyl-2-m-toly-but-3-en-2-ol (S15). 


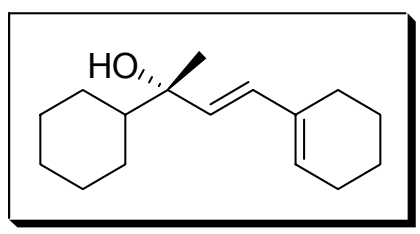

The General Procedure A was applied to cyclohexyl methyl ketone on a 69 $\mu \mathrm{L}(0.5 \mathrm{mmol})$ scale. The crude product was purified by column chromatography on deactivated silica gel $\left(\mathrm{Et}_{3} \mathrm{~N} / \mathrm{SiO}_{2}=2.5 \% \mathrm{~V} / \mathrm{V}\right.$, hexanes : EtOAc / $96: 4)$ to give the product (102 $\mathrm{mg}, 87 \%$ yield, $77 \%$ ee) as an oil: $[\alpha]_{\mathrm{D}}{ }^{20}=+1.4\left(c 2.45, \mathrm{CHCl}_{3}\right) ;{ }^{1} \mathrm{H}$ NMR $\left(\mathrm{C}_{6} \mathrm{D}_{6}, 500 \mathrm{MHz}\right) \delta 0.97-1.06(\mathrm{~m}$, $4 \mathrm{H}), 1.09-1.16(\mathrm{~m}, 1 \mathrm{H}), 1.20(\mathrm{~s}, 3 \mathrm{H}), 1.27-1.29(\mathrm{~m}, 1 \mathrm{H}), 1.45-1.49(\mathrm{~m}, 2 \mathrm{H})$, 1.51-1.55 (m, 2H), 1.70-1.73 (m, 2H), 1.82-1.85 (m, 2H), 2.00-2.01 (m, 2H), 2.04-2.07 (m, 2H), $5.61(\mathrm{~d}$, $J=16.0 \mathrm{~Hz}, 1 \mathrm{H}), 5.70(\mathrm{~m}, 1 \mathrm{H}), 6.33(\mathrm{~d}, J=16.0 \mathrm{~Hz}, 1 \mathrm{H}) \mathrm{ppm} ;{ }^{13} \mathrm{C}\left\{{ }^{1} \mathrm{H}\right\} \mathrm{NMR}\left(\mathrm{C}_{6} \mathrm{D}_{6}, 125 \mathrm{MHz}\right) \delta 22.9$, 23.0, 25.0, 26.0, 26.1, 26.9, 27.0, 27.1, 27.5, 27.9, 49.1, 74.6, 128.6, 131.3, 132.5, 135.5 ppm; IR (neat) $3448,2923,2854,1652,1449,1370,1130 \mathrm{~cm}^{-1}$; HRMS $m / z 216.1863\left[\left(\mathrm{M}-\mathrm{H}_{2} \mathrm{O}\right)^{+}\right.$; calcd for $\mathrm{C}_{16} \mathrm{H}_{24}$ : 216.1878].

\section{Preparation of 1-cyclohex-1-enyl-3,4-dimethyl-pent-1-en-3-ol (S16).}

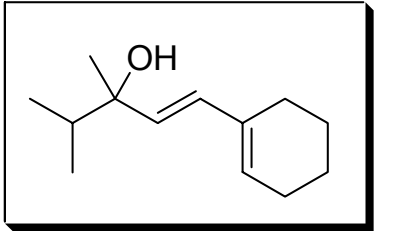

The General Procedure A was applied to 3-methyl-butan-2-one on a $54 \mu \mathrm{L}(0.5$ mmol) scale. The crude product was purified by column chromatography on deactivated silica gel $\left(\mathrm{Et}_{3} \mathrm{~N} / \mathrm{SiO}_{2}=2.5 \% \mathrm{~V} / \mathrm{V}\right.$, hexanes : EtOAc $\left./ 98: 2\right)$ to give the product ( $66 \mathrm{mg}, 68 \%$ yield, $42 \%$ ee) as an oil: $[\alpha]_{\mathrm{D}}{ }^{20}=+0.95(c 2.0$, $\left.\mathrm{CHCl}_{3}\right) ;{ }^{1} \mathrm{H} \mathrm{NMR}\left(\mathrm{C}_{6} \mathrm{D}_{6}, 500 \mathrm{MHz}\right) \delta 0.91(\mathrm{t}, J=6.2 \mathrm{~Hz}, 6 \mathrm{H}), 1.16(\mathrm{~s}, 3 \mathrm{H})$, $1.44-1.48(\mathrm{~m}, 2 \mathrm{H}), 1.51-1.55(\mathrm{~m}, 2 \mathrm{H}), 1.62-1.67(\mathrm{~m}, 1 \mathrm{H}), 1.99-2.00(\mathrm{~m}, 2 \mathrm{H})$, 2.03-2.05 (m, 2H0, $5.60(\mathrm{~d}, J=16.0 \mathrm{~Hz}, 1 \mathrm{H}), 5.69(\mathrm{t}, J=3.9 \mathrm{~Hz}, 1 \mathrm{H}), 6.33(\mathrm{~d}, J=16.0 \mathrm{~Hz}, 1 \mathrm{H}) \mathrm{ppm}$; ${ }^{13} \mathrm{C}\left\{{ }^{1} \mathrm{H}\right\}$ NMR $\left(\mathrm{C}_{6} \mathrm{D}_{6}, 125 \mathrm{MHz}\right) \delta 17.4,17.8,22.9,23.0,25.0,25.8,26.1,38.7,75.0,128.7,131.6$, 132.0, 135.5 ppm; IR (neat) 3440, 2962, 2923, 2870, 1713, 1662, $1451 \mathrm{~cm}^{-1}$; HRMS m/z 176.1567 [(M$\left.\mathrm{H}_{2} \mathrm{O}\right)^{+}$; calcd for $\mathrm{C}_{13} \mathrm{H}_{20}$ : 176.1565].

\section{General Procedure B. Preparation of 4-methyl-2-phenyl-pent-3-en-2-ol (S17).}

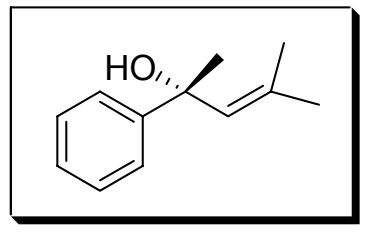

The ligand 1 (27.3 mg, $10 \mathrm{~mol} \%)$ was weighed into the well-dried Schlenk flask and loaded into the drybox. The bis(2-methyl-1-propenyl)zinc reagent (140 $\mathrm{mg}, 0.8 \mathrm{mmol}$ ) was weighed under $\mathrm{N}_{2}$ and combined with $2 \mathrm{~mL}$ toluene, followed by titanium(IV) isopropoxide $(0.43 \mathrm{~mL}, 0.6 \mathrm{mmol}, 1.4 \mathrm{M}$ toluene solution) in drybox. The reaction mixture was stirred at room temperature for 10 min and actophenone $(59 \mu \mathrm{L}, 0.5 \mathrm{mmol})$ was added neat. The flask was sealed and removed from the drybox. The reaction mixture was stirred at r.t. until TLC showed completion of the ketone. The reaction was quenched with $\mathrm{H}_{2} \mathrm{O}$, extracted into $\mathrm{CH}_{2} \mathrm{Cl}_{2}$, dried with $\mathrm{MgSO}_{4}$ and concentrated under reduced pressure. The crude product was purified by column chromatography on deactivated silica gel $\left(\mathrm{Et}_{3} \mathrm{~N} / \mathrm{SiO}_{2}=2.5 \% \mathrm{~V} / \mathrm{V}\right.$, hexanes : EtOAc / $\left.95: 5\right)$ to the product $(84 \mathrm{mg}, 95 \%$ yield, $93 \%$ ee) as an oil: $[\alpha]_{\mathrm{D}}{ }^{20}=-5.0\left(c 1.17, \mathrm{CHCl}_{3}\right){ }^{1} \mathrm{H}$ NMR $\left(\mathrm{C}_{6} \mathrm{D}_{6}, 500 \mathrm{MHz}\right) \delta 1.39(\mathrm{~s}, 3 \mathrm{H}), 1.48(\mathrm{~s}, 3 \mathrm{H}), 1.55(\mathrm{~s}, 3 \mathrm{H})$, 1.69 (br, 1H), $5.62(\mathrm{~s}, 1 \mathrm{H}), 7.06$ (t, $J=7.4 \mathrm{~Hz}, 1 \mathrm{H}), 7.19$ (dd, $J=7.6,7.5 \mathrm{~Hz}, 2 \mathrm{H}), 7.52$ (d, $J=7.6 \mathrm{~Hz}$, $2 \mathrm{H}) \mathrm{ppm} ;{ }^{13} \mathrm{C}\left\{{ }^{1} \mathrm{H}\right\}$ NMR $\left(\mathrm{C}_{6} \mathrm{D}_{6}, 125 \mathrm{MHz}\right) \delta 19.1,26.7,34.1,73.8,125.6,126.4,128.2,133.2,136.1$, 149.8 ppm; IR (neat) 3434, 3025, 2971, 2923, 1663, 1491, 1446, $1374 \mathrm{~cm}^{-1}$; HRMS m/z 158.1091 [(M$\left.\mathrm{H}_{2} \mathrm{O}\right)^{+}$; calcd for $\left.\mathrm{C}_{12} \mathrm{H}_{14}: 158.1095\right]$.

\section{Preparation of 3-(3-chloro-phenyl)-5-methyl-hex-4-en-3-ol (S18).}

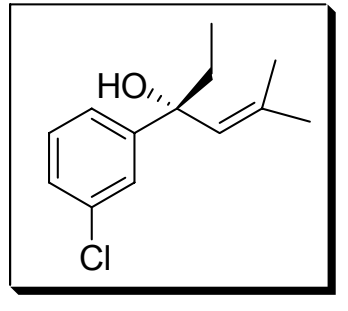

The General Procedure B was applied to 3'-chloropropiophenone on a $67 \mathrm{mg}$ $(0.4 \mathrm{mmol})$ scale. The crude product was purified by column chromatography on deactivated silica gel $\left(\mathrm{Et}_{3} \mathrm{~N} / \mathrm{SiO}_{2}=2.5 \% \mathrm{~V} / \mathrm{V}\right.$, hexanes : EtOAc / $\left.97: 3\right)$ to the product $\left(89 \mathrm{mg}, 99 \%\right.$ yield, $75 \%$ ee) as an oil: $[\alpha]_{\mathrm{D}}{ }^{20}=-1.3\left(c 0.80, \mathrm{CHCl}_{3}\right) ;{ }^{1} \mathrm{H}$ $\mathrm{NMR}\left(\mathrm{CDCl}_{3}, 500 \mathrm{MHz}\right) \delta 0.78(\mathrm{t}, J=7.4 \mathrm{~Hz}, 3 \mathrm{H}), 1.47(\mathrm{~s}, 3 \mathrm{H}), 1.73(\mathrm{~s}, 3 \mathrm{H})$, 1.75-1.80 (m, 2H), $1.83(\mathrm{br}, 1 \mathrm{H}), 5.68(\mathrm{~s}, 1 \mathrm{H}), 7.15-7.17(\mathrm{~m}, 1 \mathrm{H}), 7.19-7.22(\mathrm{~m}$, $1 \mathrm{H}), 7.27-7.28(\mathrm{~m}, 1 \mathrm{H}), 7.43(\mathrm{~s}, 1 \mathrm{H}) \mathrm{ppm} ;{ }^{13} \mathrm{C}\left\{{ }^{1} \mathrm{H}\right\} \mathrm{NMR}\left(\mathrm{CDCl}_{3}, 125 \mathrm{MHz}\right) \delta$ 8.2, 19.9, 27.2, 39.4, 76.3, 124.4, 126.4, 126.6, 129.4, 131.4, 134.2, 139.0, 150.2 ppm; IR (neat) 3475 , 2970, 2927, 1661, 1593, 1570, $1463 \mathrm{~cm}^{-1}$; HRMS m/z 206.0859 [(M- $\left.\mathrm{H}_{2} \mathrm{O}\right)^{+}$; calcd for $\mathrm{C}_{13} \mathrm{H}_{15} \mathrm{Cl}$ : 206.0862]. 
Preparation of 2, 4, 4-trimethyl-1-(2-methyl-propenyl)-cyclohex-2-enol (S19).

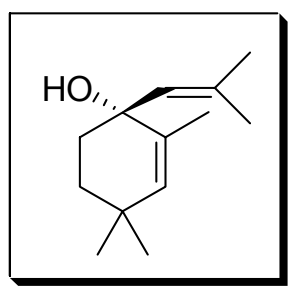

The General Procedure B was applied to 2,4,4-trimethyl-2-cyclohexen-1-one on a $60 \mu \mathrm{L}(0.4 \mathrm{mmol}) \mathrm{scale}$. The crude product was purified by column chromatography on deactivated silica gel $\left(\mathrm{Et}_{3} \mathrm{~N} / \mathrm{SiO}_{2}=2.5 \% \mathrm{~V} / \mathrm{V}\right.$, hexanes : EtOAc $\left./ 96: 4\right)$ to the product (67 mg, 86\% yield, 94\% ee) as an oil: $[\alpha]_{\mathrm{D}}{ }^{20}=-25.1\left(c 1.10, \mathrm{CHCl}_{3}\right) ;{ }^{1} \mathrm{H}$ $\operatorname{NMR}\left(\mathrm{C}_{6} \mathrm{D}_{6}, 500 \mathrm{MHz}\right) \delta 0.91(\mathrm{~s}, 3 \mathrm{H}), 0.93(\mathrm{~s}, 3 \mathrm{H}), 1.25(\mathrm{br}, 1 \mathrm{H}), 1.38-1.45(\mathrm{~m}$, $1 \mathrm{H}), 1.46-1.47(\mathrm{~m}, 1 \mathrm{H}), 1.60(\mathrm{~s}, 3 \mathrm{H}), 1.701(\mathrm{~s}, 3 \mathrm{H}), 1.703(\mathrm{~s}, 3 \mathrm{H}), 1.81-1.85(\mathrm{~m}, 1 \mathrm{H})$, $1.87-1.92(\mathrm{~m}, 1 \mathrm{H}), 5.13(\mathrm{~s}, 1 \mathrm{H}) 5.40(\mathrm{~s}, 1 \mathrm{H}) \mathrm{ppm} ;{ }^{13} \mathrm{C}\left\{{ }^{1} \mathrm{H}\right\}$ NMR $\left(\mathrm{C}_{6} \mathrm{D}_{6}, 125 \mathrm{MHz}\right) \delta$ 18.2, 18.8, 27.1, 27.9, 30.4, 31.1, 33.7, 35.6, 72.9, 131.7, 132.1, 135.3, 136.6 ppm; IR (neat) 3383, $2954,2860,1667,1447,1373 \mathrm{~cm}^{-1}$; HRMS $m / z$ 176.1561 [(M-H $\left.\mathrm{H}_{2} \mathrm{O}\right)^{+}$; calcd for $\left.\mathrm{C}_{13} \mathrm{H}_{20}: 176.1566\right]$.

\section{Preparation of 2-cyclohex-1-enyl-4-methyl-pent-3-en-2-ol (S20).}

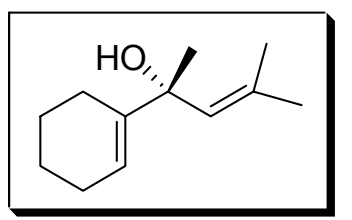

The General Procedure B was applied to 1-acetyl-1-cyclohexene on a $51 \mu \mathrm{L}$ $(0.4 \mathrm{mmol})$ scale. The crude product was purified by column chromatography on deactivated silica gel $\left(\mathrm{Et}_{3} \mathrm{~N} / \mathrm{SiO}_{2}=2.5 \% \mathrm{~V} / \mathrm{V}\right.$, hexanes : EtOAc / $\left.96: 4\right)$ to the product (50 mg, 70\% yield, $96 \%$ ee) as white solid: mp. $56-58.5{ }^{\circ} \mathrm{C} ;[\alpha]_{\mathrm{D}}{ }^{20}=+4.7$ (c 1.20, $\left.\mathrm{CHCl}_{3}\right) ;{ }^{1} \mathrm{H} \mathrm{NMR}\left(\mathrm{CDCl}_{3}, 500 \mathrm{MHz}\right) \delta 1.37(\mathrm{~s}, 3 \mathrm{H}), 1.52-1.56(\mathrm{~m}, 2 \mathrm{H})$, 1.58-1.62 (m, 2H), $1.69(\mathrm{~s}, 3 \mathrm{H}), 1.69(\mathrm{~s}, 3 \mathrm{H}), 1.97-2.00(\mathrm{~m}, 2 \mathrm{H}), 2.03-2.06(\mathrm{~m}, 2 \mathrm{H}), 5.31(\mathrm{~s}, 1 \mathrm{H}), 5.78-$ $5.79(\mathrm{~m}, 1 \mathrm{H}) \mathrm{ppm} ;{ }^{13} \mathrm{C}\left\{{ }^{1} \mathrm{H}\right\} \mathrm{NMR}\left(\mathrm{CDCl}_{3}, 125 \mathrm{MHz}\right) \delta 18.7,22.8,23.6,25.4,25.6,27.3,29.8,75.3$, 120.1, 131.2, 135.0, 143.4 ppm; IR (neat) $\mathrm{cm}^{-1}$; HRMS m/z $162.1410\left[\left(\mathrm{M}-\mathrm{H}_{2} \mathrm{O}\right)^{+}\right.$; calcd for $\mathrm{C}_{12} \mathrm{H}_{18}$ : 162.1409].

\section{1-Cyclopropyl-2-(2,4,4-trimethyl-cyclohex-2-enylidene)-ethanol (S21).}

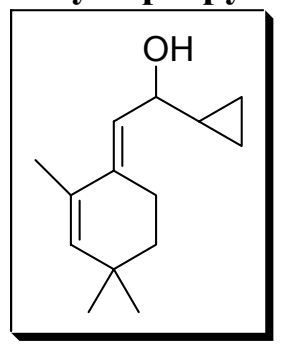

${ }^{1} \mathrm{H}$ NMR $\left(\mathrm{C}_{6} \mathrm{D}_{6}, 500 \mathrm{MHz}\right) \delta 0.28-0.36(\mathrm{~m}, 4 \mathrm{H}), 0.89(\mathrm{~s}, 3 \mathrm{H}), 0.95(\mathrm{~s}, 3 \mathrm{H}), 1.35-1.46$ $(\mathrm{m}, 3 \mathrm{H}), 1.79(\mathrm{~s}, 3 \mathrm{H}), 2.26-2.28(\mathrm{~m}, 1 \mathrm{H}), 2.36-2.40(\mathrm{~m}, 1 \mathrm{H}), 4.00(\mathrm{dd}, J=8.1,7.1 \mathrm{~Hz}$, $1 \mathrm{H}), 5.36(\mathrm{~s}, 1 \mathrm{H}), 5.56(\mathrm{~d}, J=8.3 \mathrm{~Hz}, 1 \mathrm{H}) \mathrm{ppm} ;{ }^{13} \mathrm{C}\left\{{ }^{1} \mathrm{H}\right\} \mathrm{NMR}\left(\mathrm{C}_{6} \mathrm{D}_{6}, 125 \mathrm{MHz}\right) \delta$ 2.0, 2.7, 18.4, 20.4, 23.5, 29.0, 29.5, 32.3, 37.2, 71.2, 126.1, 130.8, 136.9, 138.7 ppm; IR (neat) 3333, 3079, 2952, 2859, 1638, 1618, $1449 \mathrm{~cm}^{-1}$; HRMS-CI $\mathrm{m} / z$ $206.1673\left[\mathrm{M}^{+}\right.$; calcd for $\left.\mathrm{C}_{14} \mathrm{H}_{22} \mathrm{O}: 206.1671\right]$.

\section{1-Cyclopropyl-3-methyl-5-phenyl-penta-2,4-dien-1-ol (S22).}

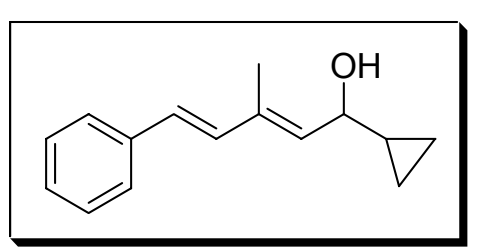
${ }^{1} \mathrm{H}$ NMR $\left(\mathrm{CDCl}_{3}, 500 \mathrm{MHz}\right) \delta 0.30-0.32(\mathrm{~m}, 1 \mathrm{H}), 0.38-0.40(\mathrm{~m}, 1 \mathrm{H})$, 0.50-0.56 (m, 1H), 0.57-0.60 (m, 1H), 1.06-1.13 (m, 1H), $1.93(\mathrm{~s}, 3 \mathrm{H})$, $4.03(\mathrm{dd}, J=8.1,8.1 \mathrm{~Hz}, 1 \mathrm{H}), 5.70(\mathrm{~d}, J=8.3 \mathrm{~Hz}, 1 \mathrm{H}), 6.58(\mathrm{~d}, J=16.1$ $\mathrm{Hz}, 1 \mathrm{H}), 6.82(\mathrm{~d}, J=16.1 \mathrm{~Hz}, 1 \mathrm{H}), 7.23(\mathrm{t}, J=7.3 \mathrm{~Hz}, 1 \mathrm{H}), 7.32(\mathrm{dd}, J=$ 7.3, $7.7 \mathrm{~Hz}, 1 \mathrm{H}), 7.42(\mathrm{~d}, J=7.7 \mathrm{~Hz}, 1 \mathrm{H}) \mathrm{ppm} ;{ }^{13} \mathrm{C}\left\{{ }^{1} \mathrm{H}\right\} \mathrm{NMR}\left(\mathrm{CDCl}_{3}\right.$, $125 \mathrm{MHz}) \delta 2.3,3.2,13.4,18.3,72.7,126.8,127.8,128.6,129.0,133.6$, 134.1, 135.9, 137.8 ppm; IR (neat) 3322, 3002, 2921, 2356, 1714, 1620, 1596, $1446 \mathrm{~cm}^{-1} ; \mathrm{HRMS} \mathrm{m/z}$ 214.1347 [ $\mathrm{M}^{+}$; calcd for $\left.\mathrm{C}_{15} \mathrm{H}_{18} \mathrm{O}: 214.1358\right]$.

\section{1-Cyclohex-2-enylidene-hexan-2-ol (S23).}

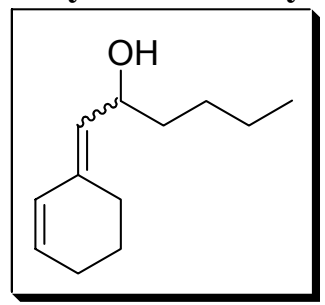
${ }^{1} \mathrm{H} \mathrm{NMR}\left(\mathrm{CDCl}_{3}, 500 \mathrm{MHz}\right) \delta 0.88(\mathrm{t}, J=12.3 \mathrm{~Hz}, 1 \mathrm{H}), 1.28-1.38(\mathrm{~m}, 3 \mathrm{H}), 1.55-$ $1.64(\mathrm{~m}, 3 \mathrm{H}), 1.77-1.81(\mathrm{~m}, 1 \mathrm{H}), 1.85-1.90(\mathrm{~m}, 1 \mathrm{H}), 2.06-2.18(\mathrm{~m}, 4 \mathrm{H}), 4.27(\mathrm{~m}$, $1 \mathrm{H}), 5.64(\mathrm{~m}, 1 \mathrm{H}), 5.66-5.72(\mathrm{~m}, 1 \mathrm{H}), 6.02(\mathrm{~d}, J=15.7 \mathrm{~Hz}, 1 \mathrm{H}) \mathrm{ppm} ;{ }^{13} \mathrm{C}\left\{{ }^{1} \mathrm{H}\right\}$ NMR $\left(\mathrm{CDCl}_{3}, 125 \mathrm{MHz}\right) \delta 14.4,19.3,22.6,25.0,32.0,32.6,32.9,66.7,128.5$, 130.7, 132.8, $139.1 \mathrm{ppm}$; IR (neat) 3344, 3021, 2919, 1623, $1454 \mathrm{~cm}^{-1}$; HRMS $m / z$ $162.1407\left[\left(\mathrm{M}-\mathrm{H}_{2} \mathrm{O}\right)^{+}\right.$; calcd for $\mathrm{C}_{12} \mathrm{H}_{18}$ : 162.1408]. 


\section{Conditions for the Determination of Enantiomeric Excess.}

The analysis of S2-3, S5-7, S10-14 and S17-18 was performed by chiral HPLC using a Chiralcel OD$\mathrm{H}$ or AS column. The conditions for the resolution are described below.

5-Phenylsulfanyl-2-(3-trifluoromethyl-phenyl)-pent-3-en-2-ol (S2). $t_{1}=36.4 \mathrm{~min}, \mathrm{t}_{2}=39.1 \mathrm{~min}$ (hexanes /2-propanol : $97 / 3,0.5 \mathrm{~mL} / \mathrm{min}, \mathrm{OD}-\mathrm{H}$ ).

3-Methyl-1-phenyl-non-4-en-1-yn-3-ol (S3). $t_{1}=16.1 \mathrm{~min}, \mathrm{t}_{2}=23.0 \mathrm{~min}$ (hexanes $/ 2$-propanol : 97 /3, $0.5 \mathrm{~mL} / \mathrm{min}, \mathrm{OD}-\mathrm{H})$.

4-Cyclohex-1-enyl-2-phenyl-but-3-en-2-ol (S5). $\mathrm{t}_{1}=15.8 \mathrm{~min}, \mathrm{t}_{2}=18.2 \mathrm{~min}$ (hexanes $/ 2$-propanol : $97 / 3,0.5 \mathrm{~mL} / \mathrm{min}, \mathrm{OD}-\mathrm{H})$.

4-Cyclohex-1-enyl-2-m-toly-but-3-en-2-ol (S6). $\mathrm{t}_{1}=15.7 \mathrm{~min}, \mathrm{t}_{2}=17.9 \mathrm{~min}$ (hexanes $/ 2$-propanol : 97 / 3, $0.5 \mathrm{~mL} / \mathrm{min}, \mathrm{OD}-\mathrm{H})$.

4-Cyclohex-1-enyl-2-(3-trifluoromethyl-phenyl)-but-3-en-2-ol (S7). $\mathrm{t}_{1}=13.9 \mathrm{~min}, \mathrm{t}_{2}=16.6 \mathrm{~min}$ (hexanes /2-propanol : 97 / 3, $0.5 \mathrm{~mL} / \mathrm{min}, \mathrm{OD}-\mathrm{H}$ ).

5-Methyl-2-phenyl-hexa-3, 5-dien-2-ol (S10). $\mathrm{t}_{1}=60.1 \mathrm{~min}, \mathrm{t}_{2}=64.6 \mathrm{~min}$ (hexanes $/ 2$-propanol : $99 /$ $1,0.2 \mathrm{~mL} / \mathrm{min}, \mathrm{OD}-\mathrm{H})$.

5-Methyl-2-(3-trifluoromethyl-phenyl)-hexa-3, 5-dien-2-ol (S11). $\mathrm{t}_{1}=15.4 \mathrm{~min}, \mathrm{t}_{2}=16.8 \mathrm{~min}$ (hexanes /2-propanol : 98 / 2, $0.5 \mathrm{~mL} / \mathrm{min}, \mathrm{OD}-\mathrm{H}$ ).

2-(2-Bromo-phenyl)-4-cyclohex-1-enyl-but-3-en-2-ol (S12). $\mathrm{t}_{1}=17.0 \mathrm{~min}, \mathrm{t}_{2}=20.9 \mathrm{~min}$ (hexanes $/ 2$ propanol : 98 / 2, $0.5 \mathrm{~mL} / \mathrm{min}, \mathrm{OD}-\mathrm{H})$.

3-(3-Chloro-phenyl)-1-cyclohex-1-enyl-pent-1-en-3-ol (S13). $\mathrm{t}_{1}=19.1 \mathrm{~min}, \mathrm{t}_{2}=20.6 \mathrm{~min}$ (hexanes /2-propanol : 98 / 2, $0.5 \mathrm{~mL} / \mathrm{min}, \mathrm{OD}-\mathrm{H})$.

3-(4-Chloro-phenyl)-1-cyclohex-1-enyl-pent-1-en-3-ol (S14). $\mathrm{t}_{1}=14.6 \mathrm{~min}, \mathrm{t}_{2}=16.2 \mathrm{~min}$ (hexanes /2-propanol : 98 / 2, $0.5 \mathrm{~mL} / \mathrm{min}, \mathrm{OD}-\mathrm{H})$.

4-Methyl-2-phenyl-pent-3-en-2-ol (S17). $\mathrm{t}_{1}=14.2 \mathrm{~min}, \mathrm{t}_{2}=15.2 \mathrm{~min}$ (hexanes /2-propanol : $99.2 /$ $0.8,0.5 \mathrm{~mL} / \mathrm{min}, \mathrm{AS})$.

3-(3-Chloro-phenyl)-5-methyl-hex-4-en-3-ol (S18). $\mathrm{t}_{1}=15.6 \mathrm{~min}, \mathrm{t}_{2}=17.5 \mathrm{~min}$ (hexanes $/ 2$-propanol : 99.5 / 0.5, $0.5 \mathrm{~mL} / \mathrm{min}, \mathrm{OD}-\mathrm{H})$.

The allylic alcohols S1, S4, S8-9, S15-S16 and S19-20 were analyzed by chiral capillary GC. The specifications for the GC analyses were as follows: Fused silica chiral capillary column (Supelco $\beta$-Dex 120): $30 \mathrm{~m} \times 0.25 \mathrm{~mm}$ (id) $\times 0.25 \mu \mathrm{m}$ film thickness. Carrier gas: nitrogen. Inlet temperature: $250{ }^{\circ} \mathrm{C}$. Detector: FID, $270{ }^{\circ} \mathrm{C}$. The conditions for the resolution of the racemates by GC are given below.

2,2-Dimethyl-propionic acid 4-hydroxy-4-phenyl-pent-2-enyl ester (S1). $\mathrm{t}_{1}=57.9 \mathrm{~min}, \mathrm{t}_{2}=60.2$ $\min \left(160^{\circ} \mathrm{C}, 1.0 \mathrm{~mL} / \mathrm{min}\right)$. 
2-Bromo-1-hex-1-enyl-cyclohex-2-enol (S4). $\mathrm{t}_{1}=32.3 \mathrm{~min}, \mathrm{t}_{2}=33.4 \mathrm{~min}\left(145^{\circ} \mathrm{C}, 1.0 \mathrm{~mL} / \mathrm{min}\right)$.

6-Methyl-2-m-tolyl-dodeca-3, 5-dien-2-ol (S8). . $\mathrm{t}_{1}=134.3 \mathrm{~min}, \mathrm{t}_{2}=137.4 \mathrm{~min}\left(170^{\circ} \mathrm{C}, 1.0\right.$ $\mathrm{mL} / \mathrm{min})$.

6-Methyl-2-(3-trifluoromethyl-phenyl)-dodeca-3, 5-dien-2-ol (S9). $\mathrm{t}_{1}=153.6 \mathrm{~min}, \mathrm{t}_{2}=157.5 \mathrm{~min}$ $\left(165^{\circ} \mathrm{C}, 1.0 \mathrm{~mL} / \mathrm{min}\right)$.

4-Cyclohex-1-enyl-2-m-toly-but-3-en-2-ol (S15). $\mathrm{t}_{1}=119.1 \mathrm{~min}, \mathrm{t}_{2}=124.3 \mathrm{~min}\left(150^{\circ} \mathrm{C}, 1.0 \mathrm{~mL} / \mathrm{min}\right)$. 1-Cyclohex-1-enyl-3,4-dimethyl-pent-1-en-3-ol (S16). $\mathrm{t}_{1}=46.7 \mathrm{~min}, \mathrm{t}_{2}=48.5 \min \left(135^{\circ} \mathrm{C}, 1.0\right.$ $\mathrm{mL} / \mathrm{min})$.

2, 4, 4-Trimethyl-1-(2-methyl-propenyl)-cyclohex-2-enol (S19). $\mathrm{t}_{1}=5.0 \mathrm{~min}, \mathrm{t}_{2}=5.2 \mathrm{~min}\left(165^{\circ} \mathrm{C}\right.$, $1.0 \mathrm{~mL} / \mathrm{min})$.

2-Cyclohex-1-enyl-4-methyl-pent-3-en-2-ol (S20). $\mathrm{t}_{1}=39.1 \mathrm{~min}, \mathrm{t}_{2}=40.3 \mathrm{~min}\left(115^{\circ} \mathrm{C}, 1.0 \mathrm{~mL} / \mathrm{min}\right)$.

\section{Determination of the Absolute Configuration}

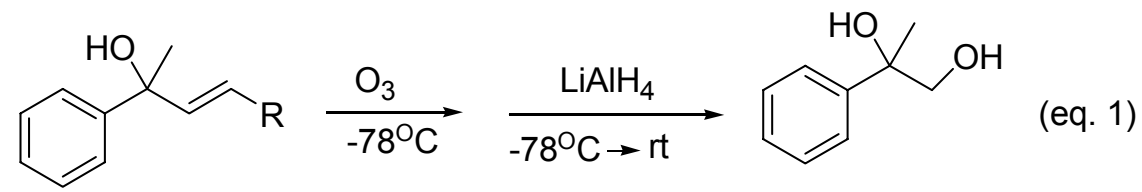

(S)-2-phenyl-oct-3-en-2-ol (entries 1 and 2 in Table 2) Determined with the products by further transformation in eq. $1 .^{3}$

$$
[\alpha]_{\mathrm{D}}^{20}=-6.95\left(c 2.0, \mathrm{Et}_{2} \mathrm{O}\right) ; \text { lit. }^{4}(\mathrm{~S}) \text {-diol: }[\alpha]_{\mathrm{D}}{ }^{20}=8.94\left(c 6.8, \mathrm{Et}_{2} \mathrm{O}\right) .
$$

The absolute configuration of the product diol should have the opposite assignment compared to the starting compound; therefore, a diol with configuration (R) will be produced from an allylic alcohol of absolute configureation (S).

(S)-4-cyclopropyl-2-phenyl-but-3-en-2-ol (entry 3 in Table 2) Determined with the products by further transformation in eq. $1^{3}$

$[\alpha]_{\mathrm{D}}{ }^{20}=-8.33\left(c 2.1, \mathrm{Et}_{2} \mathrm{O}\right)$; lit. ${ }^{4}(\mathrm{~S})$-diol: $[\alpha]_{\mathrm{D}}{ }^{20}=8.94\left(c\right.$ 6.8, $\left.\mathrm{Et}_{2} \mathrm{O}\right)$.

(S)-5-(tert-butyl-diphenyl-silanyloxy)-2-phenyl-pent-3-en-2-ol (entries 4 and 5 in Table 2)

Determined with the products by further transformation in eq. $1^{3}$

$[\alpha]_{\mathrm{D}}{ }^{20}=-9.20\left(c\right.$ 1.0, $\left.\mathrm{Et}_{2} \mathrm{O}\right)$; lit. ${ }^{4}(\mathrm{~S})$-diol: $[\alpha]_{\mathrm{D}}{ }^{20}=8.94\left(c 6.8, \mathrm{Et}_{2} \mathrm{O}\right)$.

(S)-2,4-diphenyl-but-3-en-2-ol (entries 6 and 7 in Table 2) Determined with the products by further transformation in eq. $1^{3}$

$[\alpha]_{\mathrm{D}}{ }^{20}=-5.33\left(c 0.6, \mathrm{Et}_{2} \mathrm{O}\right)$; lit. ${ }^{4}(\mathrm{~S})$-diol: $[\alpha]_{\mathrm{D}}{ }^{20}=8.94\left(c 6.8, \mathrm{Et}_{2} \mathrm{O}\right)$.

(S)-2,2-dimethyl-propionic acid 4-hydroxy-4-phenyl-pent-2-enyl ester (entries 8 and 9 in Table

2) Determined with the products by further transformation in eq. $1^{3}$ 
$[\alpha]_{\mathrm{D}}{ }^{20}=-6.53\left(c 1.7, \mathrm{Et}_{2} \mathrm{O}\right)$; lit. ${ }^{4}(\mathrm{~S})$-diol: $[\alpha]_{\mathrm{D}}{ }^{20}=8.94\left(c 6.8, \mathrm{Et}_{2} \mathrm{O}\right)$.

(S)-5-methyl-2-phenyl-hexa-3, 5-dien-2-ol (entry 1 in Table 4) Determined with the products by further transformation in eq. $1^{3}$

$[\alpha]_{\mathrm{D}}{ }^{20}=-7.42\left(c 1.2, \mathrm{Et}_{2} \mathrm{O}\right)$; lit. ${ }^{4}(\mathrm{~S})$-diol: $[\alpha]_{\mathrm{D}}{ }^{20}=8.94\left(c 6.8, \mathrm{Et}_{2} \mathrm{O}\right)$.

(S)-4-cyclohex-1-enyl-2-phenyl-but-3-en-2-ol (entry 2 in Table 4) Determined with the products by further transformation in eq. $1^{3}$

$[\alpha]_{\mathrm{D}}{ }^{20}=-6.80\left(c 0.5, \mathrm{Et}_{2} \mathrm{O}\right)$; lit. ${ }^{4}(\mathrm{~S})$-diol: $[\alpha]_{\mathrm{D}}{ }^{20}=8.94\left(c 6.8, \mathrm{Et}_{2} \mathrm{O}\right)$.

(S)-4-methyl-2-phenyl-pent-3-en-2-ol (entries 1 and 2 in Table 5) Determined with the products by further transformation in eq. $1^{3}$

$[\alpha]_{\mathrm{D}}^{20}=-6.86\left(c 1.4, \mathrm{Et}_{2} \mathrm{O}\right)$; lit. ${ }^{4}(\mathrm{~S})$-diol: $[\alpha]_{\mathrm{D}}{ }^{20}=8.94\left(c 6.8, \mathrm{Et}_{2} \mathrm{O}\right)$.

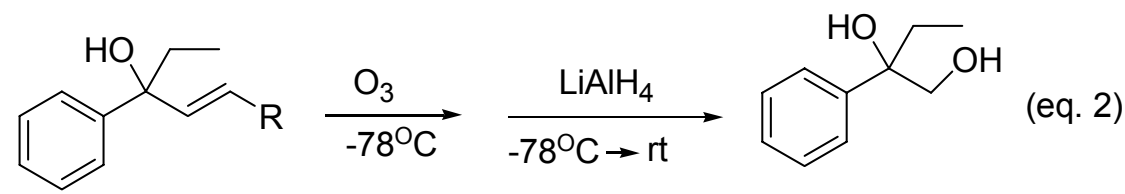

(S)-3-phenyl-non-4-en-3-ol (entry 17 in Table 2) Determined with the products by further transformation in eq. $2^{3}$

$$
[\alpha]_{\mathrm{D}}{ }^{20}=+10.23(c 2.2, \mathrm{EtOH}) ; \text { lit. }^{5,6}(\mathrm{~S})-\mathrm{diol}:[\alpha]_{\mathrm{D}}{ }^{20}=-11.4(c 3.7, \mathrm{EtOH}) .
$$

All the above absolute configuration of the compound was based on the (R,R)-ligand 1 ; the compounds with opposite absolute configuration can also be prepared using (S,S)-ligand $\mathbf{1}$.

\section{Reference:}

1. Buchwald, S. L.; LaMaire, S. J.; Neilsen, R. B.; Watson, B. T.; King, S. M. Tetrahedron Lett. 1987, 28, 3895-3898.

2. Buchwald, S. L.; LaMaire, S. J.; Neilsen, R. B.; Watson, B. T.; King, S. M. Organic Synthesis 1993, 71, 77-82.

3. Mahler, H.; Braun, M. Chem. Ber. 1991, 124. 1379-1395.

4. Eliel, E. L.; Freeman, J. P. J. Am. Chem. Soc. 1952, 74, 923-928.

5. Agami, C.; Couty, F.; Lequesne, C. Tetrahedron 1995, 51, 4043-4056.

6. Mukaiyama, T.; Sakito, Y.; Asami, M. Chem. Lett. 1978, 1253-1256. 